\title{
Desenvolvimento de um sistema de execução de manufatura (MES) no planejamento e controle de produção: uma aplicação da Indústria 4.0 no processo de fabricação de tubos de aço
}

\section{Development of a manufacturing execution system (MES) in production planning and control: an application of Industry 4.0 in the steel pipe manufacturing process}

\author{
Karina de Lima Souza \\ Universidade Estadual de Santa Catarina (UDESC) \\ karinalimaep@gmail.com \\ Iramar Baptistella do Nascimento \\ Universidade Estadual de Santa Catarina (UDESC) \\ iramar.nascimento@udesc.br

\section{Sandro Keine} \\ Universidade Estadual de Santa Catarina (UDESC) \\ sandro.keine@udesc.br

\section{Raquel Fleig} \\ Universidade Estadual de Santa Catarina (UDESC) \\ raquel.fleig@udesc,br
}

\section{RESUMO}

Empenhar esforços na melhoria contínua é primordial para atingir sucesso e longevidade nas organizações. Operando em ambiente competitivo permeado constantemente pela busca por baixos custos, muitas encontram na filosofia lean os recursos adequados para a desejada diferenciação. Atentando-se a tal expediente, o objetivo deste artigo foi desenvolver e implantar um sistema de gerenciamento de manufatura capaz de monitorar as etapas do processo produtivo em tempo real e de promover retroalimentação a fim de contribuir para as tomadas de decisão habituais durante a execução das obras. Abordagem qualitativa caracterizada em seus fins como uma pesquisa dedutiva, estando essa fundamentada em uma revisão bibliográfica e apoiada em um estudo de caso. Os resultados mostraram a aplicação do sistema MES em relação a rastreabilidade e ao armazenamento em nuvem. Por fim, o trabalho fornece um direcionamento para implementação do MES, a camada cyber dos sistemas ciberfísicos (CPS), considerado como um dos elementos de base para a Indústria 4.0.

Palavras-chave: Indústria 4.0. Manufatura Enxuta. Engenharia de Produção.

${ }^{*}$ RECEBIDO EM 04/05/2020. ACEITO EM 24/08/2020. 


\begin{abstract}
Committing efforts to continuous improvement is essential to achieve success and longevity in organizations. Operating in a competitive environment permeated constantly by the search for low costs, many find in lean philosophy the appropriate resources for the desired differentiation. Given this expedient, the objective of this article was to develop and implement a manufacturing management system capable of monitoring the stages of the production process in real time and promoting feedback in order to contribute to the usual decision making during the execution of the construction. Qualitative approach characterized in its ends as a deductive research, which is based on a literature review and supported by a case study. The results showed the application of the system in relation to traceability and cloud storage. Finally, the work provides guidance for implementing the MES.
\end{abstract}

Keywords: Industry 4.0. Lean Manufacturing. Production Engineering.

\title{
1 .Introdução.
}

O cenário industrial no início do século XXI foi um período pelo qual o processo de fabricação de produtos passou a ser personalizado. O que no passado se tratava apenas da produção artesanal de um único modelo, se transformou em algo muito mais versátil e complexo, e junto com essa evolução surgiu-se a necessidade de um planejamento de produção mais elaborado em um novo mercado globalmente competitivo (Oliveira, 2013). Segundo Moro e Júnior (2016) para o sucesso profissional no mercado concorrente, as empresas precisam ser ágeis e estar em constante mudanças na fabricação de novos produtos, com processos produtivos ágeis.

As organizações geralmente operam em ambientes caracterizados pela intensa competição, progresso tecnológico contínuo, novas exigências dos consumidores e recursos naturais escassos (BHASIN, 2012). Por mais de uma década, houve crescente interesse em operações mais flexíveis e mais responsivas, juntamente com redução no tempo de ciclo para melhorar o desempenho de todo o fluxo (DINESH; GHUPTA, 2005).

O controle dos processos de produção tem influência significativa no desempenho dos sistemas de manufatura (SCHOLZ-REITER; KÜCK; LAPPE 2014). Após determinar um conjunto de ordens de produção com base em pedidos de clientes e previsão de demanda, o agendamento e sequenciamento das ordens de produção em diferentes máquinas de um sistema de manufatura devem ser conduzidos de forma a otimizar os principais indicadores de desempenho (SCHUH et al., 2017). Os sistemas de manufatura estão sujeitos a efeitos dinâmicos que induzem mudanças rápidas e imprevistas, tais como ordens de alta prioridade não esperadas ou paradas não planejadas da máquina (NYHUIS, 2017).

O serviço baseado em informática tem extrema importância nos dias atuais, a ciência de transformar dados em informação é essencial para criação de valor para o cliente (LEE; KAO; YANG, 2014, SAARIJÄRVI; GRÖNROOS; KUUSELA, 2014). Para Amaral (2016) a ideia de indústria avançada cresce gradativamente utilizando as tecnologias de informação, automatização de máquinas, troca de dados em tempo real, desde o projeto até a produção dos produtos. O princípio baseado na 
introdução de interfaces digitais é o conjunto de tecnologias que interligadas torna possível a indústria 4.0. Essa comunicação entre o mundo físico e o digital é classificada em três grupos:

1) Conversão de elementos físicos em informação digital;

2) Tecnologias transmitindo informações reais para tomada de decisão;

3) Inteligência competitiva, transformando informação digital aplicada na gestão e organização.

Atualmente, as tecnologias da informação crescem rapidamente com o avanço e a extensão dos recursos da computação. Esse crescimento afeta vários campos, que consomem essas tecnologias. A automação industrial não é uma exceção, referindo um processo de informações gerais e flexível para implementar a função Manufacturing Execution System (MES), que pode ser implantada no ambiente industrial.

Neste contexto, este trabalho tem como propósito desenvolver e implantar um sistema de gerenciamento de manufatura capaz de monitorar as etapas do processo produtivo em tempo real e de promover retroalimentação a fim de contribuir para as tomadas de decisão habituais durante a execução das obras em uma empresa de fabricação de tubos de aço localizada em SC. A utilização do conceito lean e tecnologias modernas a fim de reduzir o custo e identificar possíveis melhorias foram essenciais para a realização do projeto, aproveitando a capacidade de obter um sistema de manufatura que encaminhe informações de imediato do chão de fábrica ao escritório, direcionando as tomadas de decisão para um padrão mais eficaz.

\section{Desenvolvimento.}

A filosofia lean surgiu após a segunda guerra mundial, com a aplicação de suas principais ferramentas estritamente destinadas a área fabril, com o principal objetivo de combater os desperdícios. $\mathrm{O}$ lean office segue os mesmos princípios do Lean Manufacturing para a área administrativa da empresa, mesmo sem contemplar produção de itens físicos, os escritórios utilizam recursos como tempo e conhecimento, entregando informações ao cliente de forma clara e rápida (NETLAND, 2016).

Alves, Dinis e Souza (2012) retratam a importância da produção enxuta como modo pensar e desenvolver melhorias contínuas, enfrentando as necessidades de mudanças no mercado atual, tornando as empresas mais ágeis. A aplicação do lean manufacturing vem crescendo gradativamente nos últimos anos, onde algumas empresas que aderiram ao sistema de produção enxuta demonstram resultados satisfatórios, em contrapartida, demais empresas não obtiveram o mesmo êxito, sendo um dos motivos a falta de entendimento e compreensão de todos os envolvidos no processo.

Na implantação do lean manufacturing é necessário a compreensão de todo o sistema de produção e sua estrutura, visualizar amplamente o fluxo de informações e materiais, determinando assim, as práticas a serem admitidas (LIMA et al., 2016). Para Schulze e Störmer (2012) mesmo que o lean dentro da indústria tenha conquistado o sucesso, a implementação ainda é novidade ou está em processo de transição nas empresas. Além disso, muitas empresas acham difícil sustentar o ímpeto inicial de seu projeto lean na manufatura (NETLAND, 2016).

O conceito lean office foi desenvolvido a partir do Lean Thinking, aplicados inicialmente no chão de fábrica, no entanto, sua técnica e princípios básicos são aplicáveis em qualquer parte de uma empresa, até mesmo nos processos administrativos (CAVAGLIERI, 2015).

A aplicação bem realizada na produção permitiu a adaptação das ferramentas enxutas nos setores administrativos, na praticidade do gerenciamento do banco de dados, redução de desperdícios no fluxo 
de informações, tempo de espera do cliente, eliminação de procedimentos que geram resíduos (KURIGER, 2010). Almeida et al. (2017) comentam sobre a abordagem do lean office que possibilita reduzir a superprodução de documentos impressos, otimizando o tempo para fornecer serviços, reduzindo movimentações excessivas de pessoas no local, minimizando os custos de armazenamentos.

Para Campos et al. (2016) a manufatura enxuta sempre teve como foco principal a identificação de possíveis melhorias nos processos, por ser visual e tangível, já o fluxo de informação não era considerado como âmbito de aplicações. A facilidade de mapear processos nas organizações ganhou credibilidade e melhorou significativamente no século XXI. Isso foi possível de duas maneiras: primeiro, usando o software de computador visualmente para exibir gráficos, dados e a interação de ações, pessoas e os níveis relevantes de autoridade e também responsabilidades; e segundo, através da ampla acessibilidade dos mapas de processos nas organizações, via internet, intranet e conectividade móvel (ROWELL, 2018). Embora o uso de mapas de processo e mapeamento para o desempenho organizacional seja relativamente amplo, os benefícios alcançados pelas organizações que o adotam são apenas parcialmente compreendidos.

Por outro lado, há pesquisas sobre o uso e aplicação da modelagem de processos, mas grande parte é baseada nos aspectos técnicos (notação e gramática) dos sistemas de modelagem. Vom Brocke et al. (2014) afirmam que a pesquisa se concentra principalmente na modelagem de processos, sistemas de fluxo de trabalho e fatores críticos de sucesso. Embora as operações no nível de negócios e no nível da planta estejam relacionadas e dependentes umas das outras, os dois níveis são frequentemente apenas vagamente integrados, com troca lenta de informações (por exemplo, em tempo não real e não automatizada) entre os dois. Além disso, os esforços para integrar sistemas de negócios de nível superior com controle do lado da fábrica podem ser prejudicados pela necessidade de código de programação especializado, exigindo o envolvimento de programadores experientes ou pessoal de TI que possam ter um entendimento limitado das operações do lado do negócio e do lado da planta em comparação com a gerência ou o pessoal da fábrica (CORONADO, 2018).

A tecnologia da informação surgiu por volta da década de 1950, designado como um sistema de aplicação do computador e equipamentos de telecomunicações para armazenar, recuperar, transmitir e manipular dados, ou seja, ferramentas que viabilizam a comunicação eficiente, muitas vezes no contexto entre empresas diferentes (DAINTITH, 2012). Segundo Teeravaraprug e Inklay (2014) as tecnologias da informação foram categorizadas em seis grupos: comunicação, transação, planejamento, gerenciamento de organização e gerenciamento de transporte.

A disponibilidade de dados de uma operação de fabricação pode ser usada para permitir um aumento na capacidade, adaptabilidade e conscientização do processo. Nos sistemas ciber-físicos atuais, os dados são coletados de peças de equipamento de fabricação e usados para gerar mudanças úteis e afetar a produção. Os dados coletados geralmente descrevem o estado operacional do equipamento, como uma máquina-ferramenta, e podem ser fornecidos usando protocolos padrão (CORONADO, 2018).

Para o desempenho global das empresas é essencial o controle das informações, tratar, proteger e gerar dados para a cadeia de valor, utilizando meio de recursos como: processos, pessoas, tecnologia, rede e dados (Luo; Cheng, 2006; Pinto, 2008). Existem dois grupos importantes de sistema de informação (SI), os sistemas de suporte à operação e o sistema de suporte à gestão.

A gestão da produção tem função fundamental de controlar os fluxos físicos, compilando informações para serem executadas e suportadas no fluxo de informação, que vão desde comunicação direta entre pessoas, até arquivos técnicos e documentos. Esse sistema e ferramentas implementados, permitem o melhor controle de produção e, consequentemente, redução de custos da organização, tornando a acessibilidade em tempo real, com informações que fluem desde os colaboradores da empresa até a gestão de topo, permitindo que todas pessoas transmitam as informações necessárias de forma relevante (COURTOIS; PILLET; MARTIN-BONNEFOUS, 2010). 
Ao longo da década de 60 foram desenvolvidas diversas tecnologias para um controle de produção mais eficiente, tais como o Materials Requirements Planning (MRP), ferramenta considerada poderosa para evidenciar problemas e corrigi-los. Com a principal função de assegurar que os materiais utilizados para a produção, foram planejados na quantidade correta (sem desperdícios), com qualidade exigida (zero defeitos), no tempo certo e local certo (COURTOIS; PILLET; MARTIN-BONNEFOUS, 2010).

Em seguida, através do MRP deu-se origem ao Enterprise Resources Planning (ERP), com aumento de eficiência para manter um posicionamento competitivo e com a rápida evolução, esse sistema foi projetado para integrar e otimizar processos na empresa, no qual, também incluía clientes e fornecedores (BABAEI; GHOLAMI; ALTAFI, 2015). Portanto o ERP, utiliza o planejamento de recursos com um sistema de dados integrados aos processos do chão de fábrica, porém englobando toda a gestão dos fluxos envolvidos, caracterizado por três níveis: estratégico, tático e operacional (ADDOTENKORANG; HELO, 2011).

Os benefícios da execução de um sistema ERP são intangíveis, podendo citar como exemplo a visibilidade da empresa, otimização no processo, resposta rápida ao cliente, melhoria na comunicação interna da organização, integração entre os sistemas, flexibilidade nos dados e rastreabilidade de informações (SHEN; CHEN; WANG, 2016). Babaei, Gholami e Altafi (2015) comentam que a dificuldade da implantação do ERP, caso o resultado não seja favorável, não está no software e sim na complexidade de informações que passaram por mudanças, estão contidas para que o sistema flua continuamente.

A Indústria 4.0 baseia-se na integração de tecnologias de informação e comunicação que permitem alcançar novos patamares de produtividade, flexibilidade, qualidade e gerenciamento, possibilitando a geração de novas estratégias e modelos de negócio para a indústria, sendo, por isso, considerada a Quarta Revolução Industrial. Em uma planta industrial operando com Indústria 4.0, a linha de produção pode ser acionada remotamente. Para facilitar, um modelo virtual da linha de produção é criado, de modo que otimizações da linha de produção possam ser ensaiadas primeiro no computador, no mundo virtual, para garantir que, quanto implantado, venha a causar o mínimo de problemas possível (SACOMANO et al., 2018).

Sacomano et al. (2018) apresentam os sistemas ciber físicos (CPS), internet das coisas (IoT) e internet de serviços (IoS) como sendo os elementos considerados de bases para a Indústria 4.0. As aplicações que utilizam as arquiteturas de sistemas ciber físicos (CPS) são formadas por duas camadas: camadas de tecnologia operacional (física). E camada virtual, de aplicações de tecnologia de informação (cyber). Nos CPS, a integração entre as camadas física e cyber dá-se a partir de sensores e atuadores. A camada cyber pode abrigar um modelo de simulação que reproduz o ambiente físico, além de incluir um conjunto de aplicações de tecnologia da informação (TI) como sistemas integrados de gestão (ERP), sistemas de acompanhamento e controle da produção (Manufacturing Execution System - MES), sistemas de gestão do ciclo de vida do produto (PLM), entre outros.

Uma solução completa do Manufacturing Execution System (MES) é utilizada para conexão do sistema ERP com as máquinas já automatizadas, efetuada através de um controlador lógico programável (CLP), no qual a finalidade principal é gerenciar a execução do que foi planejado, devendo disponibilizar todos os relatórios necessários para discussões e tomadas de decisão das informações em tempo real. Contudo, grande parte dos especialistas em SI não designava o sistema focado ao gerenciamento das atividades de produção da fábrica. As empresas começaram a sentir necessidade da criação de um software, que estabelecesse ligação direta entre o planejamento e o chão de fábrica (SAENZ; ARTIBA; PELLERINE, 2009).

Os sistemas de coleta de dados de produção tem o objetivo principal de controlar e monitorar os processos simultaneamente em tempo real. Neste caso, os dados para análise se tornam um pouco mais complexos, considerando o grande número de aplicações que vieram com o tempo (SAENZ; ARTIBA; PELLERINE, 2009). No intuito do desenvolvimento de soluções mais simples e integradas foi criado MES, fornecendo uma interface comum, e um sistema de gestão unificado. 
O conceito MES se originou por volta dos anos 90, com a necessidade das empresas em atender as exigências do mercado, no quesito qualidade, produzindo conforme normas estabelecidas, redução de custos e cumprimento aos prazos. A partir disso, esse sistema começou a ter sucesso nas empresas devido a rastreabilidade dos seus produtos (NAEDELE et al., 2015). O sistema MES é classificado entre o nível intermediário e alto da gestão unificada de informações, proporcionando melhorar o monitoramento dos dados em trabalho de curso (Work in Progress, WIP) e recolhendo dados diretamente da fábrica.

O MES coloca em prática os planos fornecidos pelo sistema corporativo, como o ERP, no chão de fábrica. À medida que as empresas contemporâneas enfrentam o desafio representado pelo ambiente de mercado e intensas competições, torna-se cada vez mais importante que as empresas atendam rapidamente à demanda dos clientes e manter a confiabilidade de informações (JEON, 2017).

Conforme Jeon (2017) os MES foram recentemente introduzidos para monitorar vários objetos de manufatura em fábricas dinâmicas, podendo alavancar a eficiência do fluxo de informações através das camadas funcionais para planejamento e controle. No entanto, as práticas atuais do MES usando algoritmos tradicionais de posicionamento interno enfrentam várias dificuldades no rastreamento de ordens de produção e informações internas para fabricação sem fio:

As principais ações do MES incluem o planejamento, sequenciamento, envio das ordens de produção, apontamentos realizados durante a operação e coleta de informações através de dispositivos online instalados na fábrica, interligados diretamente ao SI para armazenamento de dados (WITSCH; VOGEL-HEUSER, 2012).

A organização Manufacturing Enterprise Solutions Association (MESA) relata o MES como um sistema que permite a melhoria contínua desde a emissão da ordem de produção até o produto acabado. Com o aprimoramento dos sistemas anteriores, este permite através de dados obtidos em tempo real uma rápida resposta e melhor estratégia na redução de atividades que não agregam valor ao processo (SAENZ; ARTIBA; PELLERINE, 2009).

Em uma empresa que busca incorporar a filosofia lean, produzir com qualidade exigida pelos clientes e produção, baixo custo, zero desperdício e defeito, sobrevivendo ao mercado competitivo, a comunicação entre o ERP e o MES é um fator indispensável para a compreensão da pesquisa. A indústria 4.0 se concentra no estabelecimento de produtos e processos de produção, as fábricas têm de lidar com a necessidade de desenvolvimento de produtos rápidos e produção flexível (VYATKIN et al., 2007). Conforme afirma Einsiedler (2013), uma fábrica inteligente permite a comunicação entre humanos, máquinas e produtos, capazes de adquirir e processar dados, podendo autocontrolar certas tarefas e interagir com humanos via interfaces (MA GA; HONG; SACHIN, 2011, LU, 2017).

Tendências e necessidades são atendidas para a era da Indústria 4.0. A descoberta de novas tecnologias acompanhou o desenvolvimento da indústria a partir da adoção antecipada de sistemas mecânicos, para apoiar processos de produção, até hoje altamente automatizado linhas de montagem, a fim de ser ágil e adaptável para exigências e demandas dinâmicas atuais do mercado (GORECKY et al., 2014).

Os controladores industriais e sua programação de controle associada são fundamentais para a operação dos sistemas modernos de automação industrial. Esses controladores interagem com os dispositivos de campo no chão de fábrica para realizar processos controlados relacionados a objetivos como fabricação de um produto, manuseio de materiais, processamento em lote, tratamento de águas residuais e outros processos. São armazenados e executam programas de controle definidos pelo usuário para efetuar a tomada de decisões em conexão com o processo controlado. Esses programas podem incluir, entre outros, lógica ladder, gráficos de funções sequenciais, diagramas de blocos de funções, texto estruturado ou outras plataformas. Em algumas modalidades, o MES na nuvem pode funcionar em sites e correlacionar dados de vários sistemas. Essa abordagem permite a melhoria da utilização geral do sistema ou equipamento de processo de vários locais ou sistemas (COTTYN, 2011). 
Adquirir dados precisos e confiáveis de máquinas e seus componentes é o primeiro passo no desenvolvimento de uma plataforma digital. Os dados podem ser medidos diretamente por sensores ou obtidos de controladores ou sistemas de manufatura da empresa, como ERP, MES SCM e CMM (LEE; BAGHERI; KAO, 2015).

Quanto mais software e inteligência incorporada são integrados em produtos industriais e sistemas, as tecnologias preditivas podem entrelaçar algoritmos inteligentes, transformando-se para a revolução industrial da $4^{a}$ geração. Essas tecnologias serão usadas para prever degradação do desempenho do produto e gerenciar de forma autônoma, otimizando as necessidades de serviço do produto (LEE; KAO; YANG, 2014).

Ao entender e identificar todos os processos, a tomada de decisão será cada vez mais assistida por sistemas de produção auto otimizados (SPATH, 2013). Sistemas distribuídos são capazes de produzir lotes menores e auxiliar particularmente as empresas a minimizar custos e identificar oportunidades de mercado (STICH; KOMPA; MEIER, 2011).

\section{Metodologia da Pesquisa.}

O presente trabalho utilizou métodos qualitativos por meio de pesquisa bibliográfica e estudo de caso. A técnica de estudo de caso é classificada como pesquisa descritiva, pois, de acordo com Pereira et al. (2018) esse método se caracteriza por ser avaliativo, descrevendo detalhadamente as áreas envolvidas e interação dos resultados.

O estudo de caso por meio deste trabalho, é um elemento primordial para acompanhar o processo produtivo, pois ele coleta e analisa as evidências de forma empírica (YIN, 2015). Essa coleta de dados pode ter origem de documentos, arquivos e registros, observação, entrevistas, entre outros. Quanto mais informações diversificadas forem recolhidas para consolidar a pesquisa, a discussão dos resultados será mais válida (YIN, 2015).

A abordagem da pesquisa é classificada como qualitativa, no qual o mapeamento de processos é baseado na realidade do processo escolhido, de modo que o pesquisador entenda o problema investigado. O método qualitativo é caracterizado por ser uma pesquisa que parte dos significados dos fenômenos vivenciados pelas pessoas (PEREIRA et al., 2018).

A pesquisa é caracterizada pelo método dedutivo, sendo um processo de análise de informação que faz uso da dedução para obter uma conclusão concreta a respeito de determinadas premissas (PEREIRA et al., 2018). A finalidade do projeto abordado foi de compreender a realidade atual dos sistemas de manufatura, utilizando como referência os conceitos lean, um conhecimento já aplicado que tem como objetivo explicar os fatos.

Para atingir o objetivo geral do estudo, analisou-se o fluxograma da interface digital do processo administrativo, comparado às informações da linha de produção. Visto que informações como ordem de produção e apontamentos do produto não estavam interligados com o sistema. Após caracterizar as principais etapas e atividades empregadas do estado atual e futuro, através da ferramenta lean que auxilia a mapear o fluxo dos processos, foi possível visualizar os possíveis desperdícios dispostos em cada atividade, sendo então propostas prováveis melhorias.

No que se refere aos objetivos, a pesquisa deu-se de forma exploratória sendo através da construção de hipóteses para melhorias contínuas, descrevendo fenômenos encontrados, e identificando fatores que contribuem para a estagnação dos processos. Pereira et al.(2018) reforçam que na pesquisa 
exploratória o principal objetivo é aperfeiçoar ideias ou a descoberta de intuições. Para a efetivação da pesquisa foram realizadas visitas, nas quais se obtiveram informações referentes aos processos produtivos da empresa; após análise desta realidade foram então propostas mudanças para a obtenção de melhorias.

A empresa estudada é considerada uma das maiores processadoras de aço do Brasil, com um aumento crescente de vendas, da quantidade de novos produtos e da área fabril. Com diversos tipos de segmentos, a empresa tem um amplo mix de produtos, incluindo tubos para indústria de petróleo, soluções para aplicação na indústria e construção civil e produtos que atendem a indústria automobilística. Fatores que contribuem na constante evolução da empresa, reestruturação e implementando novas tecnologias para melhorias necessárias.

Contudo, existe um enorme desejo em aperfeiçoar todos os processos e operações às boas práticas, adotando a filosofia lean, atingido resultados com menos recursos, desde o relacionamento com os fornecedores, colaboradores e principalmente os clientes.

Com esta perspectiva, foi decidido desenvolver um sistema de informação para o controle de produção. Este sistema permite acompanhar o que está acontecendo na produção em tempo real, gerando um relatório de informações retroativas, possibilitando a detecção dos erros e desvios em relação ao planejado, e assim, tomada de decisões com mais eficácia.

O aplicativo em questão refere-se geralmente ao controle industrial e, mais particularmente, ao uso de conjuntos de atividades gráficas para executar funções do Manufacturing Execution System (MES) em resposta a objetivos de negócios especificados.

O presente estudo está inserido no atual projeto da empresa que pretende melhorar o controle de produção e promover o armazenamento de ordens de produção no documento físico para o formato digital. Com isso, foi possível exibir todos os processos, o que irá contribuir para o aumento da eficiência operacional. Quanto à implementação do MES, o objetivo geral foi melhorar a gestão da empresa, disponibilizando para a organização as informações necessárias para integrar os processos envolvidos, sendo eles: planejamento, gerenciamento, qualidade, entre outras atividades. Neste contexto, a implementação do sistema demandou como características principais: precisão, velocidade e acessibilidade.

No projeto como um todo foi necessário estipular etapas, indicando início e término para alcançar o objetivo assim que o processo fosse encerrado, seguindo uma ordem correta. Para o estudo das operações foi seguida uma abordagem sequencial de seis etapas. Foram elas:

1. Exploração do ambiente industrial (esta etapa inicial implicou em entender e conhecer o funcionamento de toda a organização, o fluxo de trabalho, no estudo de caso);

2. Monitoramento e medição dos processos (em seguida, avaliar as formas de medições e apontamentos, e tudo o que engloba os seus processos, desde a chegada das ordens de produção, até a rastreabilidade das ordens);

3. Identificação dos pontos críticos (os dados coletados foram recolhidos e analisadas as falhas inerentes e pontos críticos encontrados, identificando assim, as oportunidades de melhorias no processo);

4. Implementações de soluções lean (delineando a situação, para entender o fluxograma do processo);

5. Planejamento (melhoria contínua, após identificado o problema, definir e implantar uma estratégia é essencial para eliminar o que não acrescenta valor ao processo); 
6. Impacto das soluções (a última fase incluiu analisar os impactos positivos e negativos, primordialmente, de modo a garantir que os resultados obtidos vão de encontro com o objetivo esperado).

Foi necessário implementar na fábrica o software e hardware do sistema de informação utilizado. Forouzan (2010) comenta que a comunicação de dados é realizada através de um meio de transmissão que explica a troca de informações entre dois dispositivos, podendo conter imagens, áudios, caracteres textuais, entre outros. Conforme a Figura 1 o MES será classificado em cinco níveis de macros com posicionamento de soluções estratégicas de integração. Segundo Linhas (2016) para o sucesso do projeto é essencial uma sinergia do conhecimento dos setores envolvidos, desde os operários até o administrativo, garantindo que o projeto seja amplamente válido para a organização, já que afetará todo o fluxo.

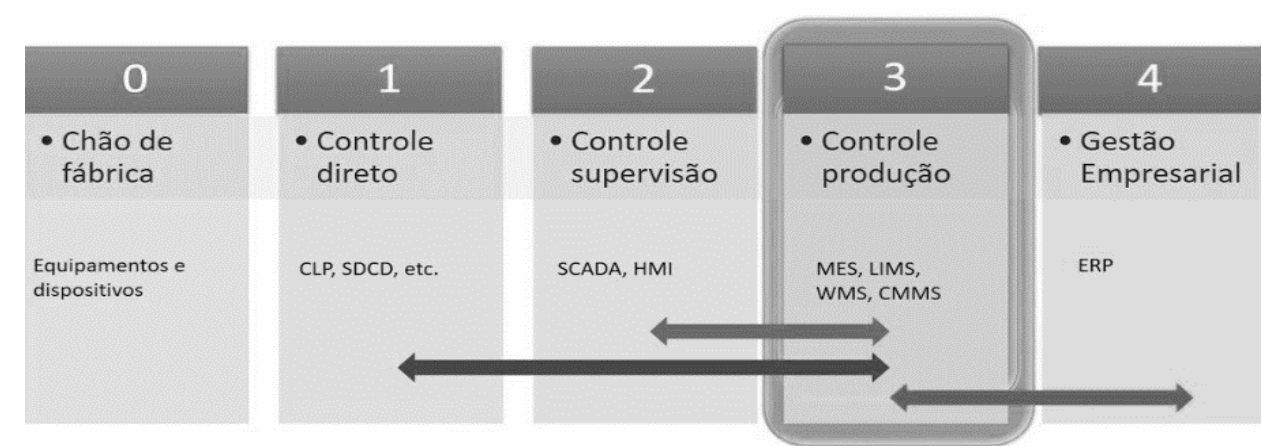

Figura 1 Integração de níveis MES.

Fonte: Autores (2019).

Durante o desenvolvimento do projeto houve a implantação de um módulo, criado dentro do ERP da empresa, especificamente para o setor de Planejamento e Controle de Produção (PCP), estudando a possibilidade de expansão para os outros setores. O sistema implementado servirá de teste piloto para futura ampliação. Com esse sistema foi possível integrar e fornecer informações desde o planejamento até o produto acabado. Além do controle e monitoramento da produção online, também permitiu que os gerentes tomassem decisões estratégicas, analisando as métricas e divulgando os resultados da qualidade, como material, não conforme, inerente e perdas. A rastreabilidade se tornou mais eficiente.

\section{Resultados e Discussão.}

O desenvolvimento do Sistema de Execução de Manufatura (MES) consiste em um sistema de interface web que desempenha juntamente com uma linguagem de programação Java. Com este sistema, é possível integrar o sistema de informação de gestão integrado com a captura de dados relacionados à fabricação, que direcionam a tomada de decisões na indústria e atendem os requisitos do Total Quality Management (TQM) considerado um sistema seguro e de fácil uso. O funcionamento do sistema trabalha conforme exemplificado conforme a Figura 2. 


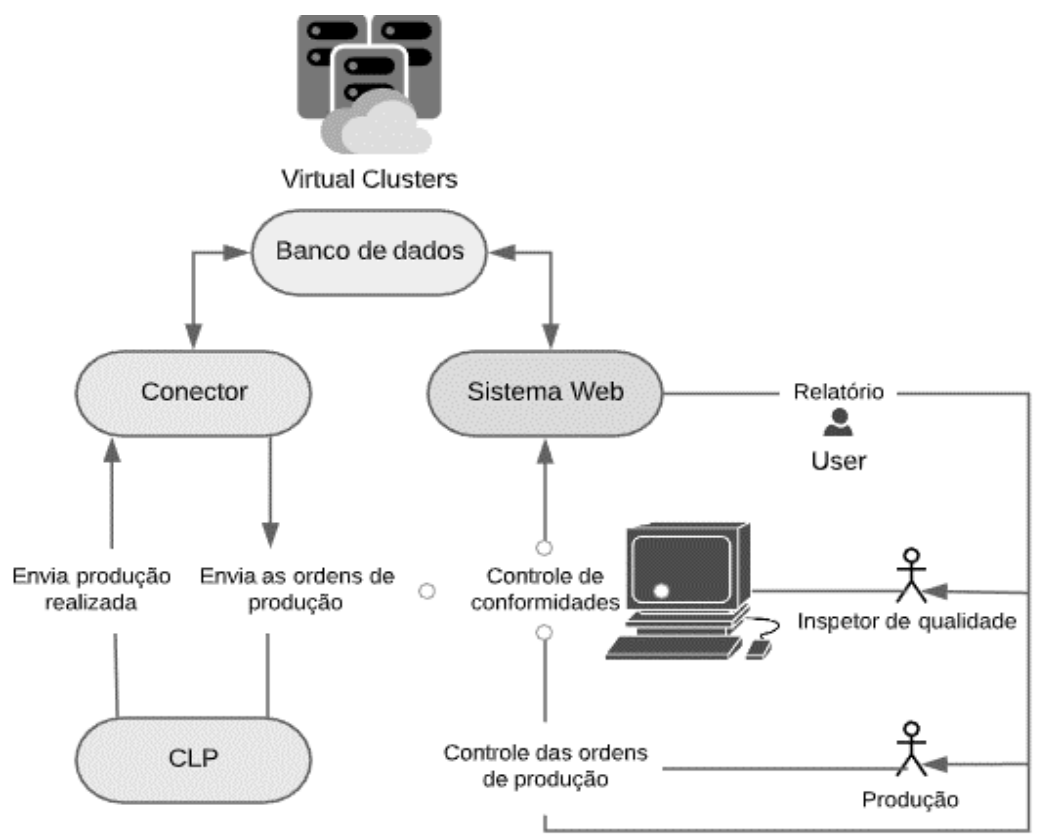

Figura 2 - Fluxograma representando o funcionamento e lógica do MES.

Fonte: Autores (2019).

Neste fluxograma (Figura 2) é possível observar que o banco de dados e o Controle Lógico Programável (CLP) fazem interligação entre o conector, exportando as informações do sistema web para o banco de dados, onde o analista tem acesso para a geração de relatórios, avaliação dos dados em tempo real, formulação de ordens de produção, etc. Na Figura 2 pode ser observada a relação entre a comunicação interna dos dados inseridos pelo chão de fábrica e a transmissão das informações no escritório, representando o uso da rede para as máquinas de modo que as monitorem-se. A Figura 2 evidencia a aplicação da arquitetura de sistemas ciber físicos (CPS), que incluem aplicações de tecnologia da informação (TI) como o Manufacturing Execution System (MES). O sistema ciber-físico reflete o plano físico do sistema de produção (instalações, produtos, máquinas e peças) no plano virtual que representa o primeiro (programas e sistemas que controlam a produção) (SACOMANO et al., 2018).

O armazenamento dos dados é realizado através de computação em nuvem, no qual os usuários possuem flexibilidade e conveniência de acessar os arquivos em qualquer local e de forma simples (ANDRADE et al., 2015). Uma das vantagens da nuvem está relacionada à sincronização automática que o sistema estabelece que, mesmo estando offline, em questão de segundos o documento é replicado. Em casos em que a sincronização não é realizada de forma automática, é chamado de hosting atuando como se fossem ficheiros carregados manualmente pelo servidor (este tipo de cloud é mais utilizado em armazenamento de "arquivo morto", sendo utilizados eventualmente).

O sistema de nuvem de computação envolveu uma unidade de armazenamento de dados configurada para gravar informações associadas a um sistema de execução de fabricação e uma interface de comunicação, para receber informações de pelo menos um site de fabricação e para interpretar as informações. Considerada uma tecnologia emergente no setor de tecnologia da informação (TI), a computação em nuvem permite a movimentação de aplicativos, serviços e dados dos computadores desktop de volta para um farm de servidores principal. O farm de servidores pode estar fora do local e ser implementado como um serviço. Ao realocar a execução de aplicativos, a implantação de serviços e o armazenamento de dados, a computação em nuvem oferece uma maneira sistemática de gerenciar custos de sistemas abertos, centralizar informações e aprimorar a robustez e reduzir os custos de energia (MCLAUGHLIN; DUCA; BURD, 2016). 
A atividade do sistema também é desenvolvida através do equipamento Sistema Digital de Controle Distribuído (SDCD), sendo o responsável pelo controle e supervisionando os processos produtivos e coletando dados tanto analógicos quanto digitais na área de produção. Um dos benefícios do SDCD é a comunicação e flexibilidade com qual qualquer hardware/software, além de possuir uma interface homem-máquina (IHM) permitindo o interfaceamento com o Controle Lógico Programável (CLP). Os dados repassados do SDCD para a interface tornando o ferramentas de gerenciamento de performance em tempo real.

Para compreender melhor o gerenciamento estabelecido pela empresa foram definidas diretrizes e estratégias da organização através do mapa de processos (Figura 3), permitindo o levantamento dos dados do fluxo de processo com o propósito de entender as etapas e integrar o ERP e chão de fábrica focando na redução de atividades que não agreguem valor, considerando os princípios do lean manufacturing e lean office.
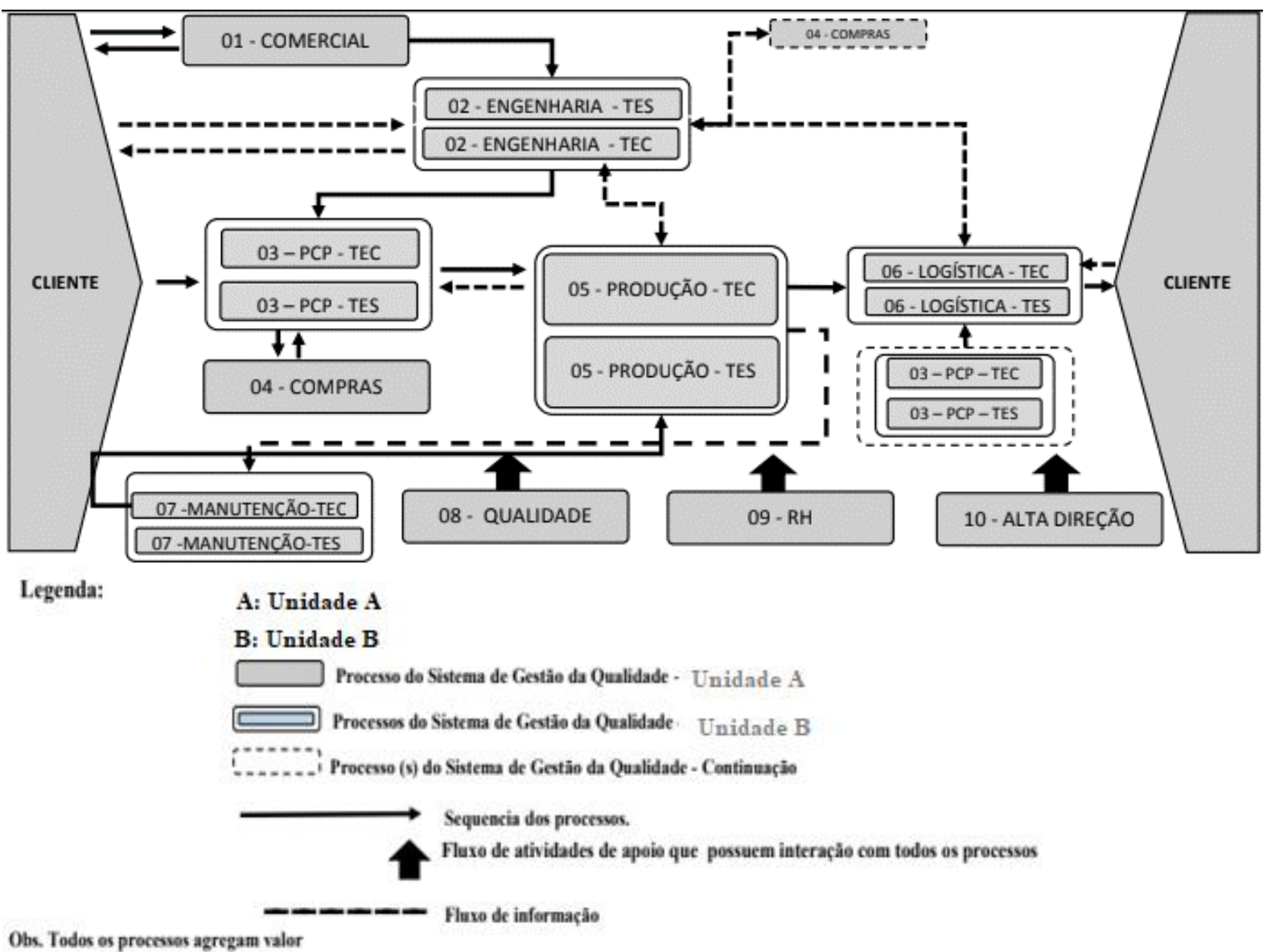

Figura 3 Fluxograma do mapeamento de processo.

Fonte: Autores (2019)

A metodologia descrita neste documento fornece uma maneira de medir o impacto e formalizar (mapear) os processos da organização, apresentando e demonstrando visualmente informações mais detalhadas do processo. Essa metodologia pode ajudar outras organizações a avaliar os estágios iniciais de seus esforços de mapeamento de processos, considerando os fundamentos do Lean Manufacturing.

Como abordado por Linhas (2016) relatou-se dificuldades em unir as informações de outras áreas envolvidas no ERP da empresa, como por exemplo, cadastros e registros de projetos que permitem manter os fluxos de trabalho através do MES, visualização e relatórios em um elemento reutilizável abrangente considerando que muitos problemas poderiam ser identificados e corrigidos de início se houvesse um envolvimento maior entre setores, implicando um grande esforço nas organizações. 
O layout e design do sistema apresentam algumas funcionalidades específicas comparado ao modelo anterior, mas com o principal objetivo de facilitar a compreensão e estimular a interação entre o usuário a um objeto digital, para que assim a informação seja recebida de forma clara e objetiva. Apresentado a um novo ideal de revolução industrial, com o objetivo de conectar e armazenar um banco de dados internos dos processos produtivos através de uma computação em nuvem, sem o gerenciamento ativo direto do usuário (MENDES, FRANZ, CAMPOS; 2017).

Para a interface gráfica no software desenvolvido foram unificados resultados secundários na tela de menu principal, tem a opção de escolha em qual ícone ou caixa de diálogo em qual o usuário deseja, organizando as categorias em: ordem de produção, testes e medições, identificação do usuário, bloqueio de tubos, inspeção visual, achatamento e expansão, testes realizados e informações gerais. Como contraponto, na barra horizontal o sistema oferece informações complementares para auxílio do operador como, gerenciador de tarefas, teclado e calculadora virtual, Figura 4.

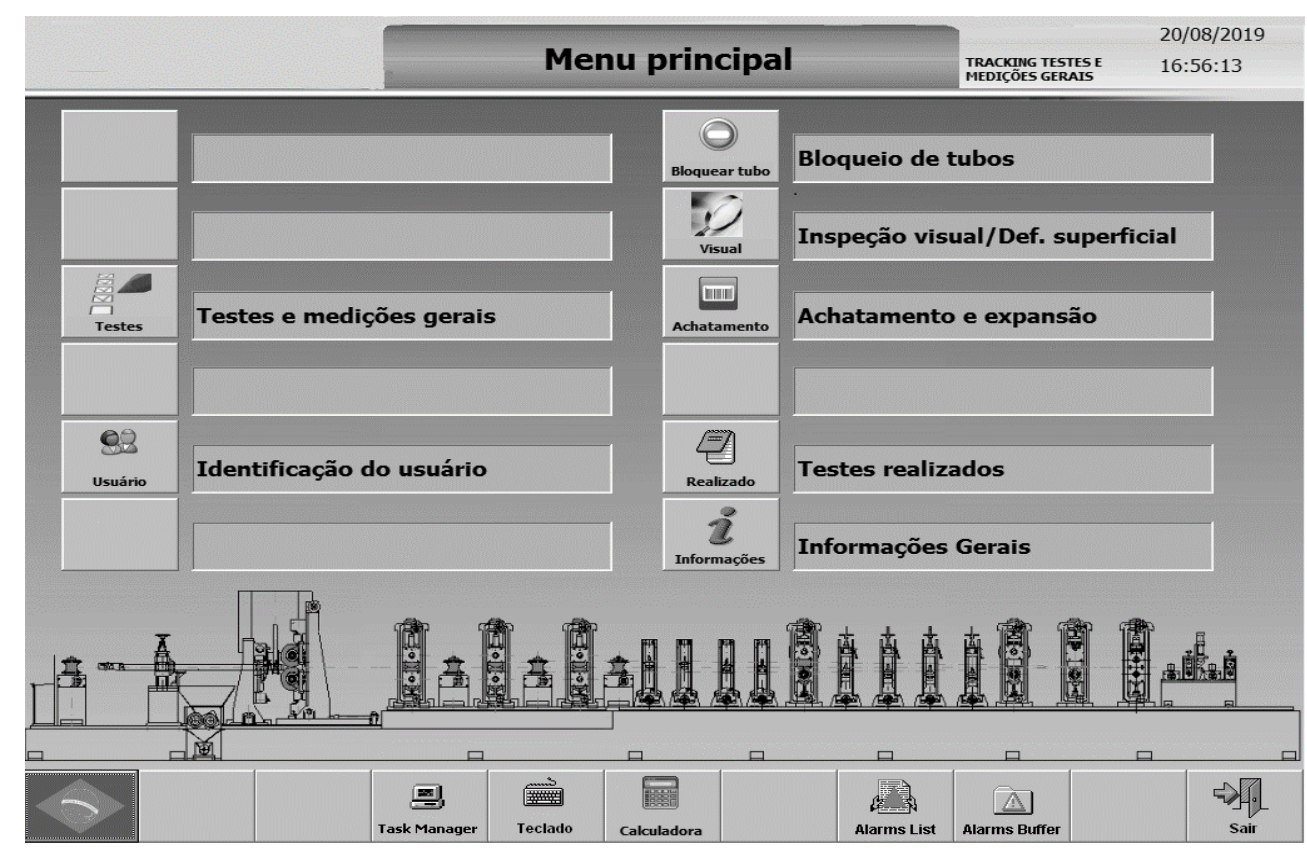

Figura 4 Representação do menu principal - Interface digital

Fonte: Autores (2019)

O layout virtual dos apontamentos e ordens de produção foi projetado a uma estrutura padrão e de fácil visualização, tornando um sistema prático e flexível. O propósito foi avaliar o desempenho do sistema em uma máquina piloto a fim de correlacionar o desenvolvimento do projeto de um processo de manufatura eficiente, determinado como Formadora 5, na justificativa em que a máquina é caracterizada por produzir itens de soldagem de chapa mais grossa que obtém um processo de conformação mais lento, de forma que a análise seja cautelosa.

É fornecido um Sistema de Execução de Fabricação (MES) que aproveita os fluxos de trabalho específicos do setor para processar solicitações de negócios. O MES pode manter conjuntos de atividades que representam fluxos de trabalho específicos do setor, que podem ser selecionados e executados para satisfazer objetivos direcionados aos negócios. Os conjuntos de atividades podem compreender operações de nível de negócios e de controle e podem ser configurados usando uma interface gráfica intuitiva que atenua a necessidade de programação de baixo nível pelo usuário final. Alguns recursos do MES são ativados ou desativados com base na compatibilidade do tipo de dados com dispositivos em comunicação com o sistema. A Figura 4 evidencia a aplicação da arquitetura de sistemas ciber físicos (CPS), que incluem aplicações de tecnologia da informação (TI) como o Manufacturing Execution System (MES). O sistema ciber-físico reflete o plano físico do sistema de 
produção (instalações, produtos, máquinas e peças) no plano virtual que representa o primeiro (programas e sistemas que controlam a produção) (SACOMANO et al., 2018).

Conforme a Figura 5 apresentada, o documento de ordem de produção em interface digital, reuniu as especificações de um item a ser produzido, incluindo descrição, quantidade a ser produzida, quantidade realizada e refugo, tempo de ciclo e padrão, favorecendo assim o planejamento e o controle da produção. Informações complementares como parada de máquina e motivo da parada também foram adicionadas ao sistema.

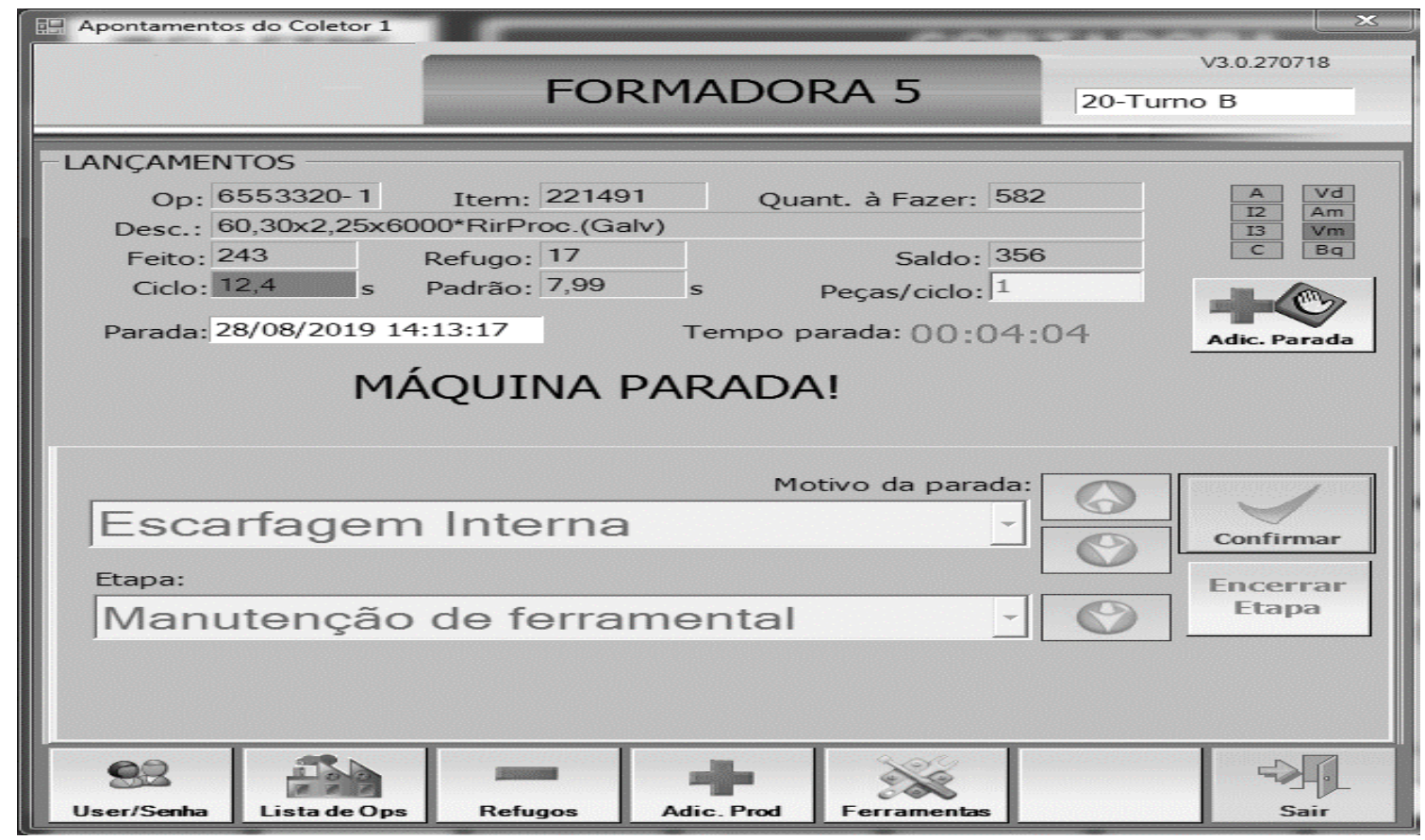

Figura 5 Representação da Interface digital de ordens de produção

Fonte: Autores (2019)

Na configuração de layout do documento das ordens de produção diferenciados pelo tipo do item que irá ser produzido há uma possibilidade para que informações e imagens complementares que precisam ser destacadas, de acordo com o item e especificações que deverão ser produzidos. Nos casos dos itens críticos o layout da tela se apresenta na cor amarela, um processo que antes já eram efetuados, porém em um documento em papel.

O sistema possui uma grande variedade de ferramentas que facilitam a integração do sistema de controle ao controle gerencial da empresa. Estas ferramentas incluem interfaces de operação, interfaces com outros dispositivos e ferramentas de engenharia.

Alguns critérios foram estabelecidos na base do sistema a fim de manter um padrão de gestão eficiente, atendendo todos os requisitos à uma possível auditoria. Estipulou-se dois critérios para análise do material com intuito de detectar o defeito ou a não conformidade. A não conformidade, corresponde a um item produzido fora do planejado, ou seja, não está dentro dos padrões ou tolerâncias normativas, já o defeito é uma falha que torna o produto inviável para comercialização. De acordo com Battisti et al. (2015) para melhor planejamento e identificação de falhas são necessários métodos e ferramentas que auxiliem na tomada de decisão da organização.

Os campos do sistema foram seguidos de acordo com um check list fundamentado na metodologia das normas ABNT 5580 e 5590, que estabelece os requisitos exigíveis para fabricação e fornecimento de tubos de aço-carbono, verificando o atendimento aos requisitos normativos, entre eles: dimensional, espessura, comprimento, grau empenamento, ortogonalidade no corte, raio, torção, esquadro, magnetismo residual, peso e informações complementares para preencher no campo de observação. Para 
Valls (2004) os princípios de gestão de qualidade são essenciais à padronização da organização mantendo sob controle os seus principais processos, gerência de recursos e equipe, baseado nas necessidades e satisfação dos clientes. Comandos como: reprovado e aprovado, em casos que o item não condiz com a tolerância normativa, simultaneamente o campo de resultado é representado pela cor vermelha (reprovado) e verde (aprovado).

Embora todas as vantagens do sistema implementado no banco de dados da gestão de produção, como rastreabilidade e informações rápidas, mesmo com a automação e mecanização, uma parte considerável continua ocorrendo tradicional, ainda é necessário atividade humana (Linhas, 2016).

Foi elaborado um sistema de gerador de relatórios com uma interface de simples visualização que possibilita a rastreabilidade dos apontamentos realizados na ordem de produção. No canto superior direito é possível observar filtros que facilitam o acesso às informações e organizam os dados obtidos, no qual podem ser filtrados através do número da ordem de produção, número da máquina ou formadora do item produzida e data do item produzido.

Para dados específicos, no canto superior esquerdo é possível observar filtros de apontamentos detalhando as medições que foram realizadas por tempo estabelecido pelo software, nos quais incluem na aba de medições gerais: comprimento, dimensional, esquadro, espessura, grau de empenamento, bisel, ortogonalidade, ovalização, raio externo e torção.

Ao buscar esses registros no banco de dados, é possível rastrear, além dos apontamentos obtidos, o dia e horário acessado, a matrícula do usuário que acessou ao software, resultado e observações comentadas pelo inspetor.

A pesquisa teve como objetivo enfatizar todas as funções imputadas ao sistema implementado, não havendo mais necessidade de acompanhamento em dados obtidos através de planilhas de excel com macros, evitando risco de erros de fórmulas e cálculos provocando informações errôneas (LINHAS, 2016).

A implementação do sistema incluiu a informatização dos documentos, que até então eram impressos e manuscritos, facilitando a integração de várias áreas da empresa. A operação do sistema é realizada em uma mesma base de dados, ou seja, todos possuem a mesma informação. Após o desenvolvimento e o sistema implementado do MES, o controle das informações aumentou, permitindo uma visão ampla da gestão, dando mais confiabilidade na tomada de decisão.

Com o uso do ERP e MES as informações passaram a ter um impacto amplo na organização, sendo necessária a sincronia com todos os setores envolvidos, desde gerência até o operacional (CAIÇARA JÚNIOR, 2008). Para Silva Júnior e Silva (2014) a nova plataforma tecnológica teve um papel importante nesse caso, permitido o desenvolvimento mais rápido de qualquer informação solicitada.

Uma das vantagens dessa abordagem é o aprimoramento do MES. Os MES são usados para fornecer instruções ou rotinas para sistemas automatizados básicos. Os sistemas automatizados básicos, por sua vez, são usados para instruir os sistemas diretamente sobre as ações a serem executadas (por exemplo, a operação real do hardware de automação).

A determinação do índice de desempenho são determinados através de medições de qualidade, eficácia, custo/benefício representando o impacto do sistema ERP na organização. Questões como: redução de custos de operação; redução de pessoal; ganhos de produtividade; aumento dos rendimentos; das vendas; nos lucros; retorno sobre o investimento; preço de estoque e qualidade do produto puderam ser percebidas no processo.

Analisou-se todo o processo de organização da indústria, desde a coleta dos documentos referidos até o seu próprio armazenamento. Verificou-se o espaço físico de $20 \mathrm{~m}^{2}$ destinado para os registros das ordens de produção, somente com arquivo morto para futura rastreabilidade, encontrando muitas ordens 
perdidas ou deterioradas. Assim, quando eram necessárias, em casos de atendimento ao cliente nas assistências técnicas ou até mesmo no produto interno, havia uma grande dificuldade em procurar o documento, ocasionando desperdício de tempo e possíveis extravios de informações.

Somando todas as dificuldades já existentes, como impressão e arquivamento e questões de sustentabilidade aliadas à tecnologia avançada, a gestão automatizada para a gestão de automação se mostrou eficiente auxiliando tanto na economia de tempo quanto de dinheiro.

A implementação do MES reforçou a segurança como firewalls associados à criptografia, trouxe como vantagem um controle rígido de acesso ao banco de dados, de modo que somente os desenvolvedores e credenciados têm determinadas informações, limitando o acesso dos usuários e reduzindo o risco de invasões. Para Forouzan (2010) e Linhas (2016) a segurança de informações e dados se tornou uma demanda atual das empresas, os meios digitais estão cada vez mais em formatos digitais, a fim de integrar as informações de sistemas, melhorando a comunicação interna, as análises dos processos internos e tomada de decisão.

Para que as empresas possam obter todos os benefícios possíveis com a implementação da automação e da Indústria 4.0 é importante que os desperdícios dos processos sejam minimizados ou eliminados (Lean Manufacturing), pois, caso os desperdícios não sejam minimizados ou eliminados do processo produtivo, as empresas correm o risco de automatizar os desperdícios, aumentando seus custos e perdendo ainda mais competitividade.

Não existem garantias de que a mudança seja vantajosa para a empresa, pois existe um temor implícito nos estudos de que é um risco grande se aventurar em tal processo e se deparar com problemas de implantação que inviabilizem o negócio. Todavia, como há um risco também em não iniciar o processo de mudança e ficar para trás. É provável que as várias modalidades de produção convivam ainda por muito tempo, de maneira que se encontre uma fábrica 4.0 de um lado da rua e do outro uma empresa 3.0 ou mesmo uma 2.0 (SACOMANO et al., 2018).

Adicionalmente, é necessário ressaltar que a Indústria 4.0 não é um modelo único, com tecnologias obrigatórias. A Indústria 4.0 se refere a um passo a mais na evolução tecnológica que pode agregar todos os aspectos organizacionais e tecnologias disponíveis. As empresas escolherão as tecnologias a serem implantadas de acordo com a sua necessidade e o retorno que esperam delas. Assim, dependendo da situação, pode ser que a empresa decida por uma mescla desse modelo com tecnologias mais antigas, mais adequadas às suas necessidades, compondo desta forma o seu modelo particular de Indústria 4.0 (SACOMANO et al., 2018).

\section{Considerações Finais.}

A aplicação de melhorias dentro de processos enxutos é um assunto que está cada vez mais presente no ambiente industrial. A busca por constante inovação, otimização dos processos, redução de custos, comprova a importância dos conceitos lean a serem empregadas em todas as etapas do trabalho.

A funcionalidade do sistema MES permitiu fornecer ainda mais informações, com mais detalhes e em tempo real, tornando a rastreabilidade dos dados mais confiáveis e informações com mais rapidez, dispensando a necessidade dos espaços de organização de ordens de produção arquivados e com custos reduzindo associados aos papéis utilizados, sendo considerada uma ferramenta de mudança a nível organizacional.

Outra vantagem foi a unificação de todas as informações no controle do Serviço de Atendimento ao Cliente (SAC), possibilitando melhoria na resposta rápida ao cliente para tomada de decisão, e a 
verificação da não conformidade dentro do processo através da rastreabilidade fornecida pelo sistema. Por fim, conclui-se que a agregação da implementação por meios eletrônicos de sistemas que controlam a produção, desde a programação do produto até o produto final, é de suma importância para que seja efetiva e cada vez mais necessária a indústria 4.0. Por aumentar o vínculo entre empresas através de cadeias de valores e banco de dados, e acompanhar este novo sistema de produção, atingindo seu potencial e benefícios a longo prazo, inovam-se a novas modalidades de controles industriais no mercado competitivo.

\section{Referências.}

ABOLLADO, J. R.; SHEHAB, E.; BAMFORTH, P. Challenges and Benefits of Digital Workflow Implementation in Aerospace Manufacturing Engineering, Procedia CIRP 60, 80-85, 2017.

ADDO-TENKORANG, R.; HELO, P. Enterprise Resource Planning (ERP): A Review Literature Report. Lecture Notes in Engineering and Computer Science, 2011.

ALVES, A.C., DINIS C., J. AND SOUZA, R.M. "Lean production as promoter of thinkers to achieve companies' agility", The Learning Organization, 19(3), 219-237, 2012.

ALMEIDA, J. P. L.; GALINA, S. V. R.; GRANDE, M. M.; BRUM, D. G. "Lean thinking: planning and implementation in the public sector", International Journal of Lean Six Sigma, 8(4), 390-410, 2017.

AMARAL, L. M. O conceito de reindustrialização, Industria 4.0 e a política industrial para o século XXI. Ciclo de debates CIP 2016-Política Industrial para o séc. XXI, 2016.

ANAND, G.; KODALI, R. Analysis of Lean manufacturing frameworks, Journal of Advanced Manufacturing Systems, (9)1, 1-30, 2010.

ANDRADE, A. P. V., DIAS, G. F., RAMOS, A. S. M., \& DE SOUZA NETO, M. V. Adoção de sistemas de armazenamento de dados na nuvem: um estudo com usuários finais, 2015.

BABAEI, M.; GHOLAMI, Z.; ALTAFI, S. Challenges of Enterprise Resource Planning implementation in Iran large organizations. Information Systems, 54, 15-27, 2015.

BATTISTI, E.; FARDIN, E. L.; CAMARGO, G. F.; SACILOTTO, G. D.; NICOLETTO, M. Redução dos custos da não qualidade de uma empresa de pequeno porte Fabricante de bases porta lâmpadas através do investimento em treinamento. 2015.

BERGAMASHI, S.; REINHARD, N., Fatores Críticos de Sucesso para Implantação de Sistemas de Gestão Empresarial, In: Sistemas ERP no Brasil (Enterprise Resource Planning) Teoria e Casos, $1^{\mathrm{a}}$ ed, Capítulo 4, São Paulo, Editora Atlas, 106-129, 2003

BHAMU, J.; SANGWAY, K. S. Lean Manufacturing: Revisão de Literatura e Questões de Pesquisa Revista Internacional de Operações e Gestão de Produção, 34 (7): 876 - 940, 2014.

BHASIN, S. Performance of Lean in large organisations. Journal of Manufacturing Systems, 2012. 
BOEHM, M.; THOMAS, O. Looking beyond the rim of one's teacup: a multidisciplinary literature review of Product-Service Systems in Information Systems, Business Management, and Engineering \& Design. Journal of Cleaner Production, 51, 245-260, 2013.

CAIÇARA JUNIOR, C. Sistemas integrados de gestão ERP: uma abordagem gerencial. 3. ed. Curitiba: Ibpex, 2008.

CAMPOS, A. T.; QUEIROZ, J. A.; LEAL, F.; YAMANE, A. K. Uma proposta de implantação dos conceitos do lean office em uma empresa do setor de autopeças. In: Encontro Nacional de Engenharia de Produção, XXXVI, João Pessoa, 2016.

CAVAGLIERI, M. Lean Archives: O emprego do Lean Office na gestão de arquivos. 2015. 202 f. Dissertação (Mestrado) - Curso de Pós-graduação em Gestão da Informação, Universidade do Estado de Santa Catarina, Florianópolis, 2015.

CHAUHAN, G.; T. SINGH. Parâmetros de medição da realização do Lean Manufacturing. Medindo a excelência nos negócios 16 (3): 57 - 71, 2012.

CORONADO, P. D. U. et al. Part data integration in the Shop Floor Digital Twin: Mobile and cloud technologies to enable a manufacturing execution system. Journal of Manufacturing Systems, v. 48, p. 25-33, 2018.

COTTYN, Johannes et al. Um método para alinhar um sistema de execução de manufatura aos objetivos do Lean. International Journal of Production Research, v. 49, n. 14, p. 4397-4413, 2011.

COURTOIS, A.; PILLET, M.; MARTIN-BONNEFOUS, C. Gestão da Produção: Para uma Gestão Industrial Ágil, Criativa e Cooperante: LIDEL, 2010.

DAINTITH, J. ed., IT, A Dictionary of Physics. Oxford University Press. 1August 2012.

DANIELSSON, C. B. An explorative review of the Lean office concept, Journal of Corporate Real Estate, 15 (3/4) 167-180, 2013.

DINESH, S.; GHUPTA, V. Application of value stream mapping for lean operations and cycle time reduction: an Indian case study. Production Planning \& Control, 16(1), 44-59, 2005.

EINSIEDLER, I. Embedded Systeme für Industrie 4.0, Product. Manag.18, 26-28, 2013.

FORBES, L.; AHMED, S. M. Modern construction: lean project delivery and integrated practices. New York: CRC Press, 2011.

FOROUZAN, B. A.; FEGAN, S. C. Protocolo TCP/IP. $3^{\text {a }}$. ed. Porto Alegre: AMGH Editora, 2010.

GONÇALVES, V. K. A.; VIANA, A. L.; MEDEIROS S. H. S. Lean Office: Estudo da Aplicabilidade do Conceito em uma Universidade Pública Federal, Espacios, 36 (18), E-1, 2015.

GORECKY, D.; SCHIMITT M.; LOSKYLL, M.; ZÜHLKE, M. Interação homem-máquina na era da indústria 4.0. $12^{\text {a }}$ Conferência Internacional IEEE de Informática Industrial (INDIN). Ieee, 2014. p. 289-294, 2014.

IMAI, M. Gemba kaizen: a commonsense approach to a continuous improvement strategy (2. ed.). New York: McGraw-Hill, 2012.

JASTI, N. K. V.; SHARMA, A. Lean manufacturing implementation using value stream mapping as a tool: a case study from auto components industry. International Journal of Lean Six Sigma, 5 (1), 89116, 2014. 
JEON, Byeong Woo et al. Um projeto de arquitetura para um sistema inteligente de execução de fabricação. Projeto e aplicações assistidas por computador, v. 14, n. 4, p. 472-485, 2017.

KUMAR, M.; ANTONY, J.; MADU, M. N.; MONTGOMERY, D. C.; PARK S. H. Common myths of Six Sigma demystified. International Journal of Quality \& Reliability Management, 25 (8), 878 895, 2008.

KURIGER, G. W.; WAN, H-d.; MIREHEI, M.; TAMMA, S.; CHEN, F. F. “A Web-Based Lean Simulation Game for Office Operations: Training the Other Side of a Lean Enterprise”, Simulation \& Gaming, 41 (4), 487-510, 2010.

LEE, J.; KAO, H.; YANG, S. Service innovation and smart analytics for industry 4.0 and big data environment. Procedia Cirp, 16, 3-8, 2014.

LEE, J.; BAGHERI, B.; KAO, H. Uma arquitetura de sistemas ciber-físicos para sistemas de manufatura baseados na indústria 4.0. Manufacturing letters, v. 3, p. 18 a 23, 2015.

LIMA, S.; ALCANTARA, P. G.; SANTOS, L. C.; SILVA, L. M. F.; DA SILVA, R. M. Mapeamento do fluxo de valor e simulação para implementação de práticas lean em uma empresa calçadista. Revista Produção Online, (16)1, 366-392, 2016.

LINHAS, O. S. Desenvolvimento e implementação de um sistema de informação para controle da produção. 2016.

LU, Y. Indústria 4.0: Uma pesquisa sobre tecnologias, aplicações e questões de pesquisa aberta. Revista de Integração da Informação Industrial, 6, 1-10, 2017.

LUO, H.; CHENG, L. The project management and the Engineering Management Information System. Beijing: China Machine Press, 2006.

MCLAUGHLIN, P. F.; DUCA, A.; BURD, M. G. Computação em nuvem para um sistema de execução de manufatura . Patente US n. 9.412.137, 9, 2016.

MA GA Y.; HONG, P.; SACHIN B.M. The impact of leanness and innovativeness on environmental and financial performance: Insights from Indian SMEs. International Journal of Production Economics, ISSN: 0925-5273, 129 (2), 251-261, 2011.

MENDES, C.; FRANZ, B. S.; CAMPOS, M. M. Estudos de caso da indústria 4.0 aplicados em uma empresa automobilística. POSGERE. São Paulo, 1, 15-25, 2017.

MONTEIRO, J.; ALVES, A. C.; CARVALHO, M. S. Processes improvement applying Lean Office tools in a logistic department of a car multimedia components company, Procedia Manufacturing 13, pp. 995-1002, 2017.

MORO, S. R., JÚNIOR, A. B. Uma revisão das abordagens para o desenvolvimento enxuto de produtos. Journal of Lean Systems, 1 (3): 91-105, 2016.

NAEDELE, M.; CHEN, H.; KAZMAN, R.; CAI, Y.; XIÃO, L.; SILVA, C. Manufacturing execution systems: a vision for managing software development. Journal of Systems and Software, 101 (1), 5968, 2015.

NALLUSAMY, S. Efficiency enhancement in CNC industry using value stream mapping, work standardization and line balancing. International Journal of Performability Engineering, v. 12, n. 5, p. 413-422, 2016. 
NAVEEN, K.; SUNIL, L.; SANJAY, K.; ABID, H. Facilitating Lean Manufacturing Systems Implementation: Role of Top Management. International Journal of Advances in Management and Economics, 2013.

NETLAND, T. H. Critical success factors for implementing lean production : the effect of contingencies. International Journal of Production Research, 2016.

NYHUIS, P.; MAYER, J. Modelling the Influence of Setup Optimized Sequencing on Lateness and Productivity Behaviour of Workstations. CIRP Annals - Manufacturing Technology, 66 (1), pp. 421424, 2017.

OLIVEIRA, T. H. Análise da aplicação dos princípios do lean manufacturing no desenvolvimento de produto: um estudo de caso na indústria eletroeletrônica.. 92 f. Dissertação (Mestrado) -Curso de PósGraduação em Engenharia de Produção, Universidade Metodista de Piracicaba, UNIMEP, Santa Bárbara D'oeste, 2013.

PEREIRA, A.S.; SHITSUKA, D.M.; FÁBIO, J.P; SHITSUKA, R. Metodologia da pesquisa científica. Brasil, 2018.

PINTO, J. P. Gestão de Operações: na Indústria e nos Serviços (2 ed.): LIDEL, 2008.

ROTHER, M.; SHOOK, J. Aprendendo a Enxergar: mapeando o fluxo de valor para agregar valor e diminuir desperdício. São Paulo: LeanInstitute Brasil, 2012.

ROWELL, J. As organizações têm uma missão para mapear processos? Revista de Gerenciamento de Processos de Negócios, 24 (1), 2-22, 2018.

SAARIJÄRVI, H.; GRÖNROOS, C.; KUUSELA, H. Reverse use of customer data: implications for service-based business models. Journal of Services Marketing, 28 (7), 529-537, 2014.

SACOMANO, J. B.; GONÇALVES, R. F.; SILVA, M. T. da; BONILLA, S. H.; SÁTYRO, W. C.; Indústria 4.0: conceitos e fundamentos. São Paulo: Blucher, 2018.

SAENZ U. B.; ARTIBA, A.; PELLERIN, R. Manufacturing execution system - a literature review. Production Planning \& Control, 20 (6), 525-539, 2009.

SCHUH, G.; REUTER, C.; PROTE, J.P.; BRAMBRING, F. J. Ays Increasing Data Integrity for Improving Decision Making in Production Planning and Control. CIRP Annals - Manufacturing Technology, 66 (1), 425-428, 2017.

SCHOLZ-REITER, B., KÜCK, B.; LAPPE, D. Prediction of Customer Demands for Production Planning - Automated Selection and Configuration of Suitable Prediction Methods. CIRP Annals Manufacturing Technology, 63 (1), 417-420, 2014.

SCHULZE, A.; STÖRMER, T. Lean product development-enabling management factors for waste elimination. International Journal of Technology Management, 57(1/2/3), 71-91, 2012.

SILVA JUNIOR, R. F.; SILVA, M. G. Implicações a partir da implementação do ERP em uma empresa do setor EPC (Engineering, Procurement and Construction). Espacios, 35 (11), 2014.

SINGH, B.; GARG, S. K.; SHARMA, S. K. Mapeamento do fluxo de valor: revisão de literatura e implicações para a indústria indiana. Revista Internacional de Tecnologia de Fabricação Avançada, 53, (5-8), 799-809, 2011. 
SHEN, Y. C.; CHEN, P. S.; WANG, C. H. A study of enterprise resource planning (ERP) system performance measurement using the quantitative balanced scorecard approach. Computers in Industry, 75, 127-139, 2016.

SPATH, D. Produktionsarbeit der Zukunft - Industrie 4.0, Stuttgart, 2013.

STICH, V.; KOMPA, S.; MEIER, C. Produktion am Standort Deutschland, 2011

SUÁREZ-BARRAZA, M., RAMIS-PUJOL, J.; ESTRADA-ROBLES, M. Applying gemba-kaizen in a multinational food company: a process innovation framework. International Journal of Quality and Service Sciences, 4 (1), 27-50, 2012.

TEERAVARAPRUG, J.; INKLAY, U. Tecnologias de Informação na Indústria de Montagem Automotiva na Tailândia. Academia Mundial de Ciências, Engenharia e Tecnologia. Revista Internacional de Engenharia Social, Comportamental, Educacional, Econômica, Comercial e Industrial, 8 (2), 507-509, 2014.

VALLS, V. M. O enfoque por processos da NBR ISO 9001 e sua aplicação nos serviços de informação. Ciência da informação, 33 (2), 2004.

VYATKIN, V.; SALSIC, Z.; ROOP, P. S.; FITZGERAKD, J. Now That's Smart!, Industrial Electronics Magazine, IEEE, 1 (4), 17-29, 2007.

VOM BROCKE, J.; SCHMEIDEL, T.; RECKER, J.; TRKMAN , P.; MARTENS, W. e VIAENE, S. Dez princípios de um bom gerenciamento de processos de negócios, Business Process Management Journal, 20 (4), 530-548, 2014.

WITSCH, M.; VOGEL-HEUSER, B. Towards a formal specification framework for manufacturing execution systems. IEEE Transactions on Industrial Informatics, 8 (2), 311-320, 2012.

WOMACK, J. P.; JONES, D. T. A mentalidade enxuta nas empresas Lean Thinking: elimine o desperdício e crie riqueza. Elsevier Editora, 2004.

YANG, T.; KUO, Y.; SU, C.T.; HOU, C.L. Lean production system design for fishing net manufacturing using lean principles and simulation optimization. Original Research Article of Manufacturing Systems, 34, 66-73, 2015.

YIN, R. K., Estudo de Caso: Planejamento e métodos. Bookman editora, 2015. 